\section{Kidney \\ Blood Pressure \\ Research}

Kidney Blood Press Res 2014;39:97-106

DOI: $10.1159 / 000355783$

Published onIIne: July 29, 2014

(C) 2014 S. Karger AG, Basel

www.karger.com/kbr

Accepted: April 11, 2014

1423-0143/14/0393-0097\$39.50/0

This is an Open Access article licensed under the terms of the Creative Commons AttributionNonCommercial 3.0 Unported license (CC BY-NC) (www.karger.com/OA-license), applicable to the online version of the article only. Distribution permitted for non-commercial purposes only.

Review

\title{
The Role of Physical Activity in the CKD Setting
}

\author{
Filippo Aucella ${ }^{a}$ Giuseppe Lucio Valente Luigi Catizone $^{b}$ \\ aNephrology and Dialysis Unit, Research Institute "Casa Sollievo della Sofferenza" Hospital, \\ San Giovanni Rotondo; 'Nephrologist, Bologna, Italy
}

\section{Key Words}

Chronic kidney disease $•$ Dialysis $•$ Physical activity

\begin{abstract}
A sedentary lifestyle contributes to the development of cardiovascular disease, hypertension, diabetes and probably cancer in the general population; this cluster of disease may be defined the diseasome of physical inactivity. Also in CKD/ESRD patients physical activity is strikingly low. As a result of growing evidence suggestive of cardiovascular benefit among the CKD population with exercise, the National Kidney Foundation recommended counseling by nephrologists to increase patients' levels of physical activity in their guideline about management of cardiovascular disease. Therefore, to maintain the well-being and functional capacity of renal patients attention should be directed toward maintaining strength and aerobic fitness as well as focusing on renal function and anemia or other comorbidities. All CKD/ESRD patients should be counseled and regularly encouraged by nephrology and dialysis staff to increase their level of physical activity.
\end{abstract}

Copyright $@ 2014$ S. Karger AG, Basel

\section{The worldwide scenario}

Although the technological revolution has been of great benefit to many populations throughout the world, it has also given an high contribution to physical inactivity (PI) and to its related worldwide epidemic of chronic diseases. Several behavioural and environmental factors, and megatrends (major forces in societal development that affect people's lives) affect population levels of physical activity (PA). Rapid urbanisation, mechanisation, and increased use of motorised transport could have caused global changes in PA. In 2009, PI was identified as the fourth leading risk factor for non-communicable diseases and accounted for more than 3 million preventable deaths [1]. So, a third of adults and four-fifths of adolescents 


\section{Kidney Blood Pressure Research}

do not reach public health guidelines for recommended levels of PA, although trend data from high-income countries suggest that occupational PA is decreasing but leisure-time PA has increased in adults [2]. Another aspect of the human movement range that has received attention is sedentary behaviour, which is usually defined as time spent sitting. Similarly to PA, sedentary behaviours occur in different domains (ie, at work, for leisure and entertainment, and while commuting).

Worldwide, $31,1 \%$ of adults are physically inactive, with proportions ranging from $17,0 \%$ in southeast Asia to about $43 \%$ in the Americas and the eastern Mediterranean. Inactivity rises with age, is higher in women than in men, and is increased in high-income countries. The proportion of 13-15-year-olds doing fewer than 60 min of PA of moderate to vigorous intensity per day is $80,3 \%$, boys being more active than girls. Continued improvement in monitoring of PA would help to guide development of policies and programs to increase activity levels and to reduce the burden of non-communicable diseases [2].

On average, physically inactive people have a life span that is 5 years shorter than that of physically active people. Moreover, the expected lifetime without long-standing illness is reduced by approximately 8 years in physically inactive people [3]. PI increases the risk of type 2 diabetes, cardiovascular disease (CVD), colon cancer, postmenopausal breast cancer, dementia and depression. These are all frequent chronic diseases associated with an enhanced risk of premature morbidity. It has recently suggested that type 2 diabetes, CVD, colon cancer, breast cancer, dementia and depression constitute a cluster of diseases, which defines a "diseasome of PI" [4]. The diseasome of PI represents diseases with highly different phenotypical presentations, but that share important pathogenetic mechanisms. Clearly, independently of body mass index (BMI), PI is a risk factor for all-cause mortality [4]. There are indications in the literature that PI is an independent cause of abdominal adiposity and a contributing factor to cognitive decline. It is possible that PI leads to the accumulation of visceral fat and consequently the activation of a network of inflammatory pathways, which not only promote the development of insulin resistance and atherosclerosis, but also lead to neurodegeneration and thereby the development of cognitive impairment [5].

The high prevalence of PI, its harmful health and environmental consequences, and the evidence of effective PA promotion strategies, make this problem a global public health priority. For the whole population, and particularly for adults, development of policies and environmental supports that increase opportunities for PA within communities would allow great progress. Interventions would probably have increased effectiveness, to the extent that they could address the determinants of PA at several levels. Ideally, PA initiatives should apply the relevant models and address the factors at individual, behavioural, social, environmental, and policy levels [6]. However, within the realities of public health practice, this ideal is difficult to achieve. Because disparities exist in amount of PA in subgroups of the populations, public health professionals need to tailor policy and environmental efforts and programs to promote increased PA opportunities everywhere, with specific attention to initiatives that address the needs of disadvantaged subgroups [6].

PA and public health is a new discipline, merging several areas of specialisation including epidemiology, exercise and sport science, behavior science, and environmental health science, among others; these different areas are needed to tackle the global pandemic of PI because multidisciplinary work is essential [7].

\section{The CKD patient}

PA levels are strikingly low in both CKD and ESRD patients [8]. Different, although small studies, overall agree showing significantly lower levels of PA compared to age-matched control individuals [9-13].

PA was measured in the Comprehensive Dialysis Study (CDS) in ambulatory dialysis patients using the so called Human Activity Profile that included Maximal and Adjusted Activity Scores, two summary measures that are indicative of PA [11]. PA was found to be 


\section{Kidney Blood Pressure Research}

Kidney Blood Press Res 2014;39:97-106

\begin{tabular}{l|l}
\hline DOI: 10.1159/000355783 & C 2014 S. Karger AG, Base
\end{tabular}

Published onlıne: July 29, 2014

www.karger.com/kbr

Aucella/Valente/Catizone: Role of Physical Activity in the CKD Setting

extremely low with scores for all age and gender categories below the 5th percentile of healthy individuals and $95 \%$ of patients had scores consonant with low fitness. Older age, female gender, diabetes, atherosclerotic disease, and a low level of education were associated with lower activity scores. Ambulatory participants in the CDS reported extremely low PA within all age and sex subgroups compared with the general population. Approximately $95 \%$ of patients had scores that corresponded to physical fitness levels below the 20th percentile for the general population. Although numerous correlates of PA were identified in this population, most are not readily modifiable. Thus, risk factor modification is unlikely to ameliorate the problem. Rather, strategies aimed to enhance participation in PA among patients on dialysis, such as education, counseling, and physical therapy, have the potential to improve fitness and self-reported functioning and should be tested prospectively.

The DOPPS study provides the first description of international patterns of exercise frequency and its association with exercise programs and clinical outcomes [13]. Exercise levels varied widely across the 12 DOPPS countries and across dialysis facilities within countries. Overall, $47.4 \%$ of participants were classified as regular exercisers, defined here as exercise equal to or more than once/week. Exercise performed once or less than once/ week was reported by $54 \%$ in the USA. Even lower exercise rates were recently reported in the United States Renal Data System Comprehensive Dialysis Study: 38\% of men and $22 \%$ of women aged $<65$ years reported walking frequently for exercise [14]. As expected, regular exercise was positively associated with ability to walk and lower BMI, and inversely associated with older age and several comorbidities. Regular exercise was also associated with better sleep quality, less severe bodily pain and better appetite [13]. In agreement with previous studies, high comorbidity burden was related to poor physical functioning, low exercise capacity and muscle wasting, so it need to be stated as a major determinant of the sedentary lifestyle of many haemodialysis patients. The DOPPS study also confirmed that mortality risk is lower for patients reporting to exercise only once a week, compared to patients exercising less than once weekly or never; and that, despite the possible benefits of exercising just once weekly, mortality risk decreased as exercise frequency increased, i.e. that the association of exercise frequency with longer survival may be dose dependent. In fact, lower mortality rates were observed in subjects who exercised daily and performing greater exercise frequency, suggesting that while any (at least once weekly) exercise is better than none, the more exercise the better [13].

Padilla et al showed that physical performance, as measured by peak oxygen consumption, 6-minute walk test, comfortable and maximal gait speed, and sit to stand test, was reduced compared with normative values among 55 patients with CKD stage 3-4 [15]. In addition, self-reported physical functioning based on the Physical Functioning scale and the Physical Component Summary (PCS) measure of the SF-36 were quite low.

The AASK trial (African American Study of Kidney Disease and Hypertension) studied patients with CKD stage 2-3 about their self-reported functioning [16]. It was found that the SF-36 PCS score was lower among these participants (43.4 \pm 10.9$)$ than population norms $(50 \pm 10)$ and was also lower among those with lower eGFR. In addition, in univariate and multivariable analysis, the PCS score was significantly higher among those who reported that they currently exercise than those who did not exercise. AASK participants reported worse physical functioning than hypertensive African Americans without CKD, but better functioning than hemodialysis patients from the HEMO study [16].

Nowadays it is quite clear that at least some aspects of physical functioning are impaired even in early stages of CKD, although the extent to which these impairments among patients with CKD are independent of age, comorbidity and PI is unclear. These factors may also mediate physical dysfunction among patients with more advanced stages of CKD, but limited data suggest that there is CKD-related dysfunction that is independent of age and inactivity. There has been almost no exploration of the potential causes or mechanisms of poor physical functioning among patients with CKD. Uremic toxins, vitamin D deficiency, chronic inflammation, hyperparathyroidism, metabolic acidosis, and anemia are among 


\section{Kidney Blood Pressure Research}

the possibilities; any or all of these could act to cause muscle wasting and/or altered neuromuscular function.

The loss of muscle mass complicates clinical syndromes and erodes patient quality of life. In patients with CKD, muscle wasting is common; it seems that the common pathway for all the metabolic derangements is related to exaggerated protein degradation relative to protein synthesis. Factors that contribute to net negative protein balance include, but are not limited to, decreased relative nutrient intake, catabolic effects of renal replacement therapies, metabolic derangements such as chronic inflammation and insulin resistance, hormonal abnormalities and concurrent morbid conditions such as frequent hospitalizations, depression, and gastroparesis [17].

So, it is quite clear that CKD has a profound negative impact on skeletal muscle function and structure leading to muscle atrophy and diminished exercise capacity. Another main factor leading to muscle wasting and sarcopenia in the CKD setting is chronic inflammation. There is now a growing body of evidence to support the notion that circulating markers of systemic inflammation are lower in individuals who regularly engage in physical activity. The CKD population are frequently sedentary and present chronic systemic inflammation; there may be great potential for long-term exercise interventions to shift habitual activity and improve health status [18].

Patients with CKD are physically inactive, often have difficulties performing activities of daily living and occupational tasks, and report a decreased health-related quality of life (HRQOL) when compared to healthy controls [15]. Exercise capacity, which is predictive of all-cause mortality and cardiovascular mortality in both health and disease, is significantly reduced in both predialysis and dialysis CKD patients. Furthermore, at the initiation of dialysis, sedentary individuals have a $62 \%$ higher risk of dying when compared with nonsedentary patients [19].

Low levels of physical activity are associated with poor functioning in this population $[20,21]$, and it is possible that PI contributes to the decline in functioning and frailty that develops over time in this group. Moreover, in general patients who reported less selfreported PA also reported poorer health-related quality of life in both the physical and mental domains, suggesting the possibility that PI could lead to impaired functioning. This possibility is biologically plausible as sedentary behavior can result in reduced physical fitness and thus in poor performance and function in the physical domain. In addition, exercise training has been shown to improve physical functioning among dialysis patients [22]. The association of lower levels of PA with lower self-reported mental health is also consistent with associations observed in the general population [7], and is potentially important as PA has the potential to relieve symptoms of depression and anxiety and improve mood [23]. Exercise performance is not only reduced in CKD stages 3 to 4 even in the absence of anemia, but exercise performance, measured by oxygen consumption or leg strength, also progressively fell as renal function deteriorated [24]. The likely causes are myriad, although, in this study, intrinsic muscle changes that result in reduced muscle strength and fatigue, and aerobic performance are likely to be dominant. To maintain the well-being and functional capacity of patients with CKD, therefore, attention should be directed toward maintaining strength and aerobic fitness as well as focusing on renal function and anemia [24].

Although there is no direct evidence that increasing PA can improve functioning or increase survival in the dialysis population, PA is a potentially modifiable risk factor for frailty, debility, and mortality. Hence, PA is distressingly low among CKD and ESRD settings. Thus, strategies to enhance activity in these patients should be explored [11].

\section{Which kind of exercise?}

Evidence from uncontrolled studies and from small randomized controlled trials shows that exercise training results in improved physical performance and functioning among patients with CKD. In addition, although there are no studies examining cardiovascular 


\section{Kidney Blood Pressure Research}

outcomes, several observations suggest that cardiovascular risk factors such as hypertension, inflammation, and oxidative stress, may be improved with exercise training in this population. Different kinds of physical activity may be proposed to CKD patients: Aerobic Exercise Training, Resistance Exercise, Combined Resistance and Aerobic Exercise [25].

In a pilot study it was clearly showed that one year of supervised aerobic exercise training significantly improves physical impairment and arterial stiffness in patients with predialysis CKD [26]. There was a change in V02peak following 12 months of exercise, comparable to the 17-23\% increase shown in other randomized studies, but values were still reduced compared to healthy sedentary populations, suggesting that disease processes may limit the extent to which improvement in V02peak can occur. As exercise capacity is an independent predictor of mortality in patients with CKD at initiation of dialysis and a $3.5 \mathrm{ml} / \mathrm{kg} / \mathrm{min}$ improvement in VO2peak is associated with decreased cardiovascular and mortality risk [27], these V02peak data suggests that early exercise interventions may reduce cardiovascular risk and improve mortality rates in this population. In the same study a trend toward deterioration in exercise capacity, endurance time, arterial stiffness and HRQOL in the control group, similar to the continued deterioration seen over time in dialysis patients was observed [20]. When the natural course of disease and sedentary aging is a decline in physical function, even maintenance of function may be considered a positive outcome. An interesting finding in this study was that even approximately $45 \mathrm{~min} /$ week of aerobic exercise for one year elicited changes in exercise capacity that may confer substantial cardiovascular and mortality benefits.

The Renal Exercise Demonstration Project [22], was unique in its large size and its focus on physical performance and health-related quality of life as the primary outcome measures rather than VO2peak. The authors were able to demonstrate that physical performance and health-related quality of life improved with exercise training and declined in those who were not assigned to the exercise interventions; the majority of the reported benefits can be ascribed to aerobic exercise training. Studies of the effects of exercise on V02peak have provided important information because they showed that patients with kidney disease could respond physiologically to exercise training in a manner that is similar to other patient groups.

Beside to aerobic exercise, it seems likely that resistance exercise training could be of benefit to these patients, and it is surprising that few studies have focused on resistance exercise training or included resistance training as part of the program [25]. Moreover, there are a few papers reporting experiences with exercise rehabilitation program that included aerobic exercise and strengthening exercise. Globally, all these papers show beneficial effect of different kind of exercises [25].

\section{Is there any risk in exercise promotion in CKD?}

The most common risk of exercise participation in the general population is musculoskeletal injury; the most serious risks are those of cardiac origin, ranging from dysrhythmia to ischemia to sudden death. The risk of both types of adverse events is higher with high-intensity exercise than with submaximal exercise. In the general population there is greater risk of adverse cardiac events for those who persist with a sedentary lifestyle than those who gradually increase their regular levels of physical activity. In comparison with risks associated with vigorous activities, risks of sudden cardiac adverse events during light and moderate intensity activities are lower.

Risk assessment and pre-participation screening should be done on an individual basis among patients with CKD as they are likely to be at higher risk of exercise-induced death than the general population but also stand to benefit more from increasing activity. There have been no reported cardiac events in any of the published exercise training studies in hemodialysis patients. Some patients tested exhibited abnormal response to exercise, such 


\section{Kidney Blood Pressure Research}

as S-T segment depression, hypertensive responses or with a hypotensive response or exhibiting significant ventricular ectopy [13]. All the abnormal exercise responses occurred in patients with known cardiac disease, and 3 also had diabetes. None was associated with adverse outcome. Beyond these studies, the risks associated with exercise in the CKD population have not been studied. Musculoskeletal risk may be increased in patients with CKD as a result of hyperparathyroidism and bone disease. Moreover, a major purpose of medical screening before exercise participation is to determine which patients are at increased risk for cardiovascular events. However, all patients with ESRD or advanced CKD are at increased risk for cardiopulmonary disease.

It is important to underline that in addition to proper medical screening, some diseasespecific considerations may reduce risk. Attention to patients' volume status and BP control is important in this population. Patients with ESRD should have their dry weight assessed frequently to avoid volume overload and may tolerate exercise best either during dialysis or on a day after a dialysis session [25]. Risk will be reduced with appropriate education of the patients about abnormal responses and symptoms and when to reduce intensity of exercise, defer exercise on a given day, stop exercise and contact the physician; prudent recommendations for starting and progressing with the program; and regular assessment of participation and responses to their program. Risk will be increased if patients remain sedentary.

\section{Relevance of physical activity promotion}

The many benefits of regular exercise for patients with CKD and ESRD may include improvements in a) physiologic measures (peak oxygen uptake), b) clinical measures (blood pressure, endothelial function, lipid profiles, and reduced inflammatory markers), c) psychological measures (fatigue, general health, anxiety, depression, vitality, and overall QOL), and d) functional measures (muscle strength, 6-minute walk test, gait speed, and sit to stand time) [28].

Considering the physiologic measures taken in account, a systematic review of 29 clinical trials showed that physical condition was significantly improved following the use of aerobic training [29]; moreover, intradialytic aerobic exercise leads to a significant increase in aerobic exercise capacity and duration of exercise [30]. After an aerobic exercise programme, muscle strength, power and fatigability in patients with advanced CKD improved significantly, indicating that aerobic exercise exerted effects comparable with those of resistance training. Resistance training also increases strength and functional capacity in patients with advanced CKD.

PA has been shown to improve blood pressure levels, lipid profile and many other cardiovascular risk factors [31]; it may also improve hemodialysis efficiency and the intradialytic phosphate removal [32]. Significant reduction in blood pressure levels has been reported after an aerobic exercise program with bicycles both in dialysis and in predialysis patients [33]. The reduction in the blood pressure can lead to a significant reduction in the number of prescribed antihypertensive drugs [34]. Among cardiovascular risk factors, inflammation is of paramount interest. Observational data suggests that habitual physical activity levels and fitness are associated with a reduced inflammatory profile and consequently improved survival. A few, small, short-duration intervention studies which increase physical fitness, strength, and activity levels have mixed effects on systemic inflammation in CKD patients; this follows similar results from short-duration studies in the general population although training in other chronic disease cohorts has demonstrated beneficial effects [35].

But the most important hard point is morbility and mortality. In this setting Roshanravan et al. reported for the first time that physical performance is associated with mortality rates [36]. They evaluated 385 ambulatory patients with stage 2-4 CKD and compared handgrip strength, usual gait speed, timed up and go (TUAG), and 6-minute walking distance with 


\section{Kidney \\ Blood Pressure Research}

\begin{tabular}{l|l}
\hline Kidney Blood Press Res 2014;39:97-106 \\
\hline DOI: $10.1159 / 000355783$ & (C) 2014 S. Karger AG, Basel
\end{tabular}

Published onlıne: July 29, 2014

www.karger.com/kbr

Aucella/Valente/Catizone: Role of Physical Activity in the CKD Setting

normative values and constructed Cox proportional hazards models and receiver operating characteristic curves to test associations with all-cause mortality. The main result of the study was that gait speed and TUAG more strongly predicted 3-year mortality than kidney function or commonly measured serum biomarkers. So, adding gait speed to a model that included estimated GFR may significantly improve the prediction of 3-year mortality. It is nowadays clear that impaired physical performance of the lower extremities is common in CKD and strongly associates with all-cause mortality [36]. Associations with mortality were similar in magnitude to kidney function and were stronger than traditionally measured biomarkers of CKD. Measurements of lower extremity function are relatively easy to perform and may capture a complex set of skeletal muscle and neurologic impairments that develop in CKD patients and substantially affect their survival. These results underline the complexity of the CKD patient and its similarity to the geriatric setting: it is well documented that physical performance in elderly individuals without CKD is significantly associated with mortality [37], and many of the same causal factors may be operative in stage 2-4 CKD.

\section{Enrolment of CKD patients in exercise programmes}

"All dialysis patients should be counseled and regularly encouraged by nephrology and dialysis staff to increase their level of physical activity (guideline 14.2)" [38]. This relevant statement was clearly reported in the 2005 publication K/DOQI Clinical Practice Guidelines for Cardiovascular Disease in Dialysis Patients; some document also stated that "evaluation of physical functioning and re-evaluation of the physical activity program should be done at least every six months (guideline 14.3b)" [38]. Moreover. the recently published UK National Institute for Health and Clinical Excellence (NICE) guidelines for early identification and management of CKD in adults in primary and secondary care include a recommendation to 'encourage people with CKD to take exercise' [39]. Although these guidelines are clearly derived on evidence for the general population and for those at high risk for cardiovascular disease [40], the common nephrological practice worldwide do not address these issues.

For example, Johansen [41] suggested that the recommendations for older adults (65 years and above) and adults aged 50-64 years with clinically significant chronic conditions and/or functional limitations, published in 2007 by the American College of Sports Medicine and the American Heart Association [42], can be applied to patients with advanced CKD. In practice this means that patients for whom exercise is not contraindicated should be instructed to start a routine of PA that is appropriate to their individual level of fitness, and safe for their clinical condition.

The training regimen should include aerobic, resistance and flexibility activities. The exercise prescriptions for HD and peritoneal dialysis patients need to be tailored individually [43]. Three basic methods of exercise programme delivery have been applied in patients with advanced CKD:

(a) a supervised outpatient program in a rehabilitation centre,

(b) a home exercise rehabilitation programme, and

(c) exercise during the HD session (intradialytic) [44].

In terms of ensuring the prescribed intensity and duration of exercise, the supervised outpatient programme is the most effective, but it is associated with the highest dropout rates [45]. For intradialytic exercise, it is safer to train during the first $2 \mathrm{~h}$ of the HD session because, after $2 \mathrm{~h}$ of dialysis, shifting of fluid from the microvasculature to the interstitium during exercise can cause a rapid reduction in relative blood volume with accompanying cardiovascular decompensation which may preclude further exercise. 


\section{Kidney \\ Blood Pressure Research}

Aucella/Valente/Catizone: Role of Physical Activity in the CKD Setting

\section{Conclusion}

There are nowadays sufficient data suggesting that exercise can improve many indicators of physical functioning, such as fitness, muscle mass, physical performance, and self-reported physical functioning in the CKD and dialysis settings. Fewer data are still now available to address cardiovascular indices, but they will probably be available very soon (Excite Study data, this Supplement [46-48]). However, yet now preliminary evidence suggests that exercise can enhance the management of hypertension, reduce inflammation, and improve endothelial function.

The optimal recommendations for exercise for patients with CKD have not been determined. However, it is reasonable to follow recommendations published by the AHA for older adults, which are also explicitly intended to be relevant to younger individuals with clinically significant chronic conditions and/or functional limitations, a description that applies to most patients with CKD [40]. As recently outlined, Exercise programmes in CKD patients should aim at safely inducing favourable physiological adaptations. Exercise planning should be scheduled at optimal volume and intensity and be based on the patient's age and comorbidities. Exercise in this high-risk population should be structured to favour compliance and cost-effectiveness [49].

\section{Disclosure Statement}

The authors of this work declare that they do not have any conflict of interests.

\section{References}

1 WHO: Global health risks: mortality and burden of disease attributable to selected major risks. Geneva: World Health Organization, 2009.

- Hallal PC, Andersen LB, Bull FC, Guthold R, Haskell W, Ekelund U; Lancet Physical Activity Series Working Group: Global physical activity levels: surveillance progress, pitfalls, and prospects. Lancet 2012;380:247257.

-3 Brønnum-Hansen H, Juel K, Davidsen M, Sørensen J: Impact of selected risk factors on expected lifetime without long-standing, limiting illness in Denmark. Prev Med 2007;45:49-53.

-4 Pedersen BK: Body mass index-independent effect of fitness and physical activity for all-cause mortality. Scand J Med Sci Sports 2007;17:196-204.

5 Pedersen BK: The Diseasome of Physical Inactivity and the role of myokines in muscle-fat cross talk. J Physiol 2009;587:5559-5568.

6 Heath GW, Parra DC, Sarmiento OL, Andersen LB, Owen N, Goenka S, Montes F, Brownson RC; Lancet Physical Activity Series Working Group: Evidence-based intervention in physical activity: lessons from around the world. Lancet 2012;380:272-281.

-7 Kohl HW $3^{\text {rd }}$, Craig CL, Lambert EV, Inoue S, Alkandari JR, Leetongin G, Kahlmeier S; Lancet Physical Activity Series Working Group: The pandemic of physical inactivity: global action for public health. Lancet 2012;380:294-305.

8 Painter P, Roshanravan B: The association of physical activity and physical function with clinical outcomes in adults with chronic kidney disease. Curr Opin Nephrol Hypertens 2013;22:615-623.

-9 Johansen KL, Chertow GM, Ng AV, Mulligan K, Carey S, Schoenfeld PY, Kent-Braun JA: Physical activity levels in patients on hemodialysis and healthy sedentary controls. Kidney Int 200;57:2564-2570.

10 Zamojska S, Szklarek M, Niewodniczy M, Nowicki M: Correlates of habitual physical activity in chronic haemodialysis patients. Nephrol Dial Transplant 2006;21:1323-1327.

11 Johansen KL, Chertow GM, Kutner NG, Dalrymple LS, Grimes BA, Kaysen GA: Low level of self-reported physical activity in ambulatory patients new to dialysis. Kidney Int 2010;78:1164-1170. 


\section{Kidney \\ Blood Pressure Research}

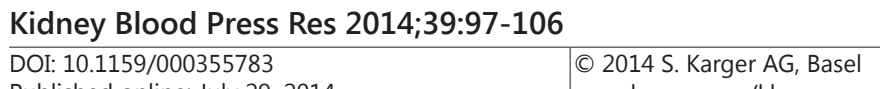

Published online: July 29, 2014

www.karger.com/kbr

Aucella/Valente/Catizone: Role of Physical Activity in the CKD Setting

12 Avesani CM, Trolonge S, Deléaval P, Baria F, Mafra D, Faxén-Irving G, Chauveau P, Teta D, Kamimura MA, Cuppari L, Chan M, Heimbürger O, Fouque D: Physical activity and energy expenditure in haemodialysis patients: an international survey. Nephrol Dial Transplant 2012;27:2430-2434.

13 Tentori F, Elder SJ, Thumma J, Pisoni RL, Bommer J, Fissell RB, Fukuhara S, Jadoul M, Keen ML, Saran R, Ramirez SP, Robinson BM: Physical exercise among participants in the Dialysis Outcomes and Practice Patterns Study (DOPPS): correlates and associated outcomes. Nephrol Dial Transplant 2010;25:3050-3062.

14 U.S. Renal Data System. U.S. Renal Data System, USRDS 2008 Annual Data Report: Atlas of Chronic Kidney Disease and End-Stage Renal Disease in the United States. Bethesda, MD: National Institutes of Health, National Institute of Diabetes and Digestive and Kidney Diseases, 2008 (Accessed December 30).

15 Padilla J, Krasnoff J, Da Silva M, Hsu CY, Frassetto L, Johansen KL, Painter P: Physical functioning in patients with chronic kidney disease. J Nephrol 2008;21:550-559.

-16 Kusek JW, Greene P, Wang SR, Beck G, West D, Jamerson K, Agodoa LY, Faulkner M, Level B: Cross-sectional study of health-related quality of life in African Americans with chronic renal insufficiency: the African American Study of Kidney Disease and Hypertension Trial. Am J Kidney Dis 2002;39:513-524.

17 Workeneh BT, Mitch WE: Review of muscle wasting associated with chronic kidney disease. Am J Clin Nutr 2010;91:1128S-1132S.

18 Dungey M, Hull KL, Smith AC, Burton JO, Bishop NC: Inflammatory factors and exercise in chronic kidney disease. Int J Endocrinol 2013;2013:569831.

19 O’Hare AM, Tawney K, Bacchetti P, Johansen KL: Decreased survival among sedentary patients undergoing dialysis: results from the dialysis morbidity and mortality study wave 2. Am J Kidney Dis 2003;41:447-454.

20 Kutner N, Zhang R, McClellan W: Patient-reported quality of life early in dialysis treatment: effects associated with usual exercise activity. Nephrol Nurs J 2000;27:357-367.

21 Johansen KL, Painter P, Kent-Braun JA, Ng AV, Carey S, Da Silva M, Chertow GM: Validation of questionnaires to estimate physical activity and functioning in ESRD. Kidney Int 2001;59:1121-1127.

22 Painter P, Carlson L, Carey S, Paul SM, Myll J: Physical functioning and health-related quality-of-life changes with exercise training in hemodialysis patients. Am J Kidney Dis 2000;35:482-492.

23 Roshanaei-Moghaddam B, Katon W, Russo J: The longitudinal effects of depression on physical activity. Gen Hosp Psychiatry 2009;31:306-315.

-24 Leikis MJ, McKenna MJ, Petersen AC, Kent AB, Murphy KT, Leppik JA, Gong X, McMahon LP: Exercise performance falls over time in patients with chronic kidney disease despite maintenance of hemoglobin concentration. Clin J Am Soc Nephrol 2006;1:488-495.

25 Johansen KL: Exercise in the end-stage renal disease population. J Am Soc Nephrol 2007;18:1845-1854.

26 Mustata S, Groeneveld S, Davidson W, Ford G, Kiland K, Manns B: Effects of exercise training on physical impairment, arterial stiffness and health-related quality of life in patients with chronic kidney disease: a pilot study. Int Urol Nephrol 2011;43:1133-1141.

-27 Sietsema KE, Amato A, Adler SG, Brass EP: Exercise capacity as a predictor of survival among ambulatory patients with end-stage renal disease. Kidney Int 2004;65:712-724.

-28 Cheema BS, Smith BC, Singh MA: A rationale for intradialytic exercise training as standard clinical practice in ESRD. Am J Kidney Dis 2005;45:912-916.

-29 Cheema BS, Singh MA: Exercise training in patients receiving maintenance hemodialysis: a systematic review of clinical trials. Am J Nephrol 2005;25:352-364.

-30 Konstantinidou E, Koukouvou G, Kouidi E, Deligiannis A, Tourkantonis A: Exercise training in patients with end-stage renal disease on hemodialysis: comparison of three rehabilitation programs. J Rehabil Med 2002;34:40-45.

-31 Kosmadakis GC, Bevington A, Smith AC, Clapp EL, Viana JL, Bishop NC, Feehally J: Physical Exercise in Patients with Severe Kidney Disease. Nephron Clin Pract 2010;115:c7-c16.

32 Orcy R, Antunes MF, Schiller T, Seus T, Böhlke M: Aerobic exercise increases phosphate removal during hemodialysis: A controlled trial. Hemodial Int 2014;18:450-458.

33 Anderson JE, Boivin MR Jr, Hatchett L: Effect of exercise training on interdialytic ambulatory and treatment-related blood pressure in hemodialysis patients. Ren Fail 2004;26:539-544.

-34 Miller BW, Cress CL, Johnson ME, Nichols DH, Schnitzler MA: Exercise during hemodialysis decreases the use of antihypertensive medications. Am J Kidney Dis 2002;39:828-833. 


\section{Kidney \\ Blood Pressure Research}

Kidney Blood Press Res 2014;39:97-106

DOI: 10.1159/000355783

Published onlıne: July 29, 2014

(C) 2014 S. Karger AG, Basel

www.karger.com/kbr

-35 Dungey M, Hull KL, Smith AC, Burton JO, Bishop NC: Inflammatory factors and exercise in chronic kidney disease. Int J Endocrinol 2013;2013:569831.

-36 Roshanravan B, Robinson-Cohen C, Patel KV, Ayers E, Littman AJ, de Boer IH, Ikizler TA, Himmelfarb J, Katzel LI, Kestenbaum B, Seliger S: Association between physical performance and all-cause mortality in CKD.J Am Soc Nephrol. 2013;24:822-830.

-37 Studenski S, Perera S, Patel K, Rosano C, Faulkner K, InzitariM, Brach J, Chandler J, Cawthon P, Connor EB, Nevitt M, Visser M, Kritchevsky S, Badinelli S, Harris T, Newman AB, Cauley J, Ferrucci L, Guralnik J: Gait speed and survival in older adults. JAMA 2011;305:50-58.

-38 K/DOQI Workgroup: K/DOQI clinical practice guidelines for cardiovascular disease in dialysis patients. Am J Kidney Dis 2005;45:S1-S53.

39 National Collaborating Centre for Chronic Conditions: Chronic Kidney Disease: National Clinical Guideline for Early Identification and Management in Adults in Primary and Secondary Care. London, Royal College of Physicians, 2008.

40 Physical Activity Guidelines Committee. Physical Activity Guidelines Advisory Committee Report, 2008. Washington, DC: U.S. Department of Health and Human services; 2008.

41 Johansen KL: Exercise and dialysis. Hemodial Int 2008;12:290-300.

42 Nelson ME, Rejeski WJ, Blair SN: Physical activity and public health in older adults:recommendation from the American College of Sports Medicine and the American Heart Association. Circulation 2007;116:10941105.

43 Fuhrmann I, Krause R: Principles of exercising in patients with chronic kidney disease, on dialysis and for kidney transplant recipients. Clin Nephrol 2004;61:s14-s25.

-44 Parsons TL, Toffelmire EB, King-VanVlack CE: Exercise training during hemodialysis improves dialysis efficacy and physical performance. Arch Phys Med Rehabil 2006;87:680-687.

-45 Kouidi E, Grekas D, Deligiannis A, Tourkantonis A: Outcomes of long-term exercise training in dialysis patients: comparison of two training programs. Clin Nephrol 2004;61:s31-s38.

46 Manfredini F, Lamberti N: Performance assessment of patient on dialysis. Kidney Blood Press Res 2014;3:176-179.

47 Baggetta R, Bolignano D, Torino C, Manfredini F, Aucella F, Barillà A, Battaglia Y, Bertoli S, Bonanno G, Castellino P, Ciurlino D, Cupisti A, D’Arrigo G, De Paola L, Fabrizi F, Fatuzzo P, Fuiano G, Lombardi L, Lucisano G, Messa P, Rapanà R, Rapisarda F, Rastelli S, Rocca-Rey L, Summaria C, Zuccalà A, Abd ElHafeez S, Tripepi G, Catizone L, Mallamaci F, Zoccali C, EXCITE Working Group: Fitness for Entering a Simple Exercise Program and Mortality: A Study Corollary to the Exercise Introduction to Enhance Performance in Dialysis (Excite) Trial. Kidney Blood Press Res 2014;3:197-204.

48 Torino C, Manfredini F, Bolignano D, Aucella F, Baggetta R, Barillà A, Battaglia Y, Bertoli S, Bonanno G, Castellino P, Ciurlino D, Cupisti A, D’Arrigo G, De Paola L, Fabrizi F, Fatuzzo P, Fuiano G, Lombardi L, Lucisano G, Messa P, Rapanà R, Rapisarda F, Rastelli S, Rocca-Rey L, Summaria C, Zuccalà A, Tripepi G, Catizone L, Zoccali C, Mallamaci F, EXCITE Working Group: Physical Performance and Clinical Outcomes in Dialysis Patients: A Secondary Analysis of the Excite Trial. Kidney Blood Press Res 2014;3:205-211.

$\$ 49$ Manfredini F, Mallamaci F, Catizone L, Zoccali C: The burden of physical inactivity in chronic kidney disease: is there an exit strategy? Nephrol Dial Transplant 2012;27:2143-2145. 